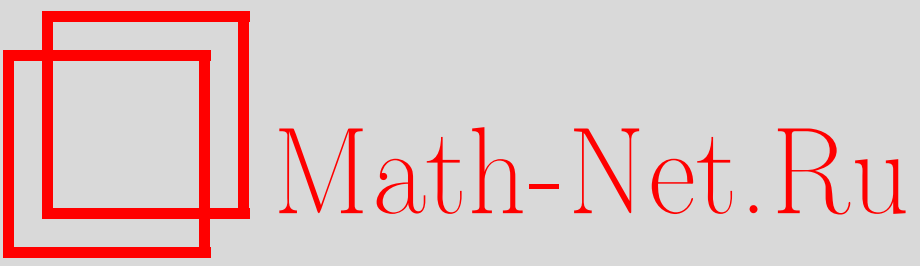

А. В. Цыганов, Об одной интегрируемой системе, связанной с шаровым волчком и цепочкой Тоды, ТМФ, 2000, том 124, номер 2, 310-322

DOI: https://doi.org/10.4213/tmf641

Использование Общероссийского математического портала Math-Net.Ru подразумевает, что вы прочитали и согласны с пользовательским соглашением

http://www.mathnet.ru/rus/agreement

Параметры загрузки:

IP : 54.157 .27 .8

26 апреля 2023 г., 13:40:47 
ТЕОРЕТИЧЕСКАЯ

И МАТЕМАТИЧЕСКАЯ

ФИЗИКА

Том 124, № 2

август, 2000

(C) 2000 г.

А. В. Цыганов*

\section{ОБ ОДНОЙ ИНТЕГРИРУЕМОЙ СИСТЕМЕ, СВЯЗАННОЙ С ШАРОВЫМ ВОЛЧКОМ И ЦЕПОЧКОЙ ТОДЫ}

\footnotetext{
Изучается интегрируемое движение по сфере $S^{2}$ в потенциале $V=\left(x_{1} x_{2} x_{3}\right)^{-2 / 3}$ с кубическим по импульсам дополнительным интегралом движения. Построено представление Лакса без спектрального параметра и рассмотрена связь с трехчастичной цепочкой Тоды.
}

\section{1. ВВЕДЕНИЕ}

Рассмотрим частный случай движения точки в пространстве $\mathbb{R}^{9}$, которое в общем случае описывается следующим уравнением Ньютона:

$$
F^{*}(t) \frac{d^{2} F(t)}{d t^{2}}+|\operatorname{det} F(t)|^{1-\gamma} G=0 .
$$

Здесь $F(t)$ и $G$ - неособые квадратные матрицы третьего порядка, $F^{*}$ - транспонированная матрица $F$ и $\gamma$ - произвольный параметр. Элементы $F_{j k}$ матрицы $F$ являются координатами данного пространства $\mathbb{R}^{9}$.

Данное уравнение возникло в теории адиабатических движений облака сжимаемого газа с однородной деформацией, в которых скорости частиц газа являются линейными функциями координат. Такие движения сплошной среды изучались в большом числе работ, первыми из которых были классические работы Дирихле, Дедекинда и Римана по теории фигур равновесия идеальной несжимаемой гравитирующей жидкости. Исследование подобных моделей продолжается до сих пор [1-5]. Построение данной физической модели и история вопроса подробно обсуждаются в книге [4].

В данной физической модели $\gamma$ - показатель адиабаты, а постоянная матрица $G$ является симметрической $G=G^{*}$. Используя канонические преобразования координат $F^{\prime}=U F U^{*}$, можно всегда привести постоянную симметрическую матрицу $G$ в уравнении (1.1) к диагональному виду $G=\operatorname{diag}\left(\varepsilon_{1}, \varepsilon_{2}, \varepsilon_{3}\right)$, где $\varepsilon_{j}= \pm 1[1]$.

\footnotetext{
${ }^{*}$ Санкт-Петербургский государственный университет, Санкт-Петербург, Россия. E-mail: tsiganov@mph.phys.spbu.ru
} 
Инвариантность системы относительно внешних и внутренних врашений газа приводит к тому, что при $G=G^{*}$ лагранжева система (1.1) инвариантна относительно шестимерной группы $S O(4) \simeq S O(3) \times S O(3)$ преобразований

$$
\mathrm{F} \rightarrow \mathrm{O}_{1} \mathrm{FO}_{2}
$$

где $O_{1}$ и $O_{2}$ - ортогональные матрицы. Вследствие этого система имеет шесть интегралов движения, образуюших две трехмерные кососимметричные матрицы

$$
\mathbf{J}=F(t) \dot{F}^{*}(t)-\dot{F}(t) F^{*}(t), \quad \mathbf{K}=F^{*}(t) \dot{F}(t)-\dot{F}^{*}(t) F(t),
$$

которые определяют полный момент количества движения и вихрь. Полная энергия газа, заполняющего эллипсоид, пропорциональна функции Гамильтона

$$
H=\sum_{i, k}^{3} \varepsilon_{k}\left(\frac{\partial F_{i k}}{\partial t}\right)^{2}-\frac{2}{\gamma-1}|\operatorname{det} F|^{1-\gamma},
$$

которая является седьмым независимым интегралом движения. Однако в обшем случае данного количества интегралов движения не хватает для того, чтобы свести решение уравнений движения к квадратурам.

Для идеального одноатомного газа с $\gamma=5 / 3$ система уравнений обладает дополнительным восьмым интегралом движения

$$
\mathbf{r}^{2}=\operatorname{tr}\left(F^{*}(t) F(t)\right)
$$

который был найден в работе [3]. Качественное поведение решений уравнений движения в зависимости от величины показателя адиабаты $\gamma$ и различные колебательные режимы расширения врашаюшегося газового облака в вакууме подробно обсуждаются в книге [4].

Уравнения движения газового эллипсоида (1.1) для диагональных и внедиагональных элементов матрищы $F$ можно рассматривать отдельно $[1,2,4]$. Шесть кинематических уравнений для внедиагональных элементов описывают внешнее и внутреннее вращения облака газа и зависят только от распределения масс газа внутри облака. Три динамических уравнения для диагональных элементов описывают расширение облака газа и зависят только от сил внутреннего давления в облаке.

Всюду далее положим $G=I, \gamma=5 / 3$ и рассмотрим расширение эллипсоидального облака газа фиксированной ориентации, т.е. при нулевом значении момента количества движения и нулевом вихре $\mathbf{J}=\mathbf{K}=0$. В этом случае исходная диагональная матрица $F_{0}(t)$ остается диагональной в любой другой момент времени

$$
F(t)=\operatorname{diag}\left(x_{1}, x_{2}, x_{3}\right)(t)
$$

Соответствующие уравнения движения в конфигурационном пространстве имеют вид

$$
x_{1} \ddot{x}_{1}=x_{2} \ddot{x}_{2}=x_{3} \ddot{x}_{3}=\frac{\text { const }}{\left(x_{1} x_{2} x_{3}\right)^{2 / 3}} \quad \text { при } \quad \gamma=\frac{5}{3} .
$$


Добавочный интеграл движения (1.3) равен $\mathbf{r}^{2}=\sum x_{k}^{2}$. Используя данный интеграл, рассмотрим вместо движения в трехмерном пространстве $\mathbb{R}^{3}$ движение по поверхности сферы $S^{2}$ радиуса $\mathbf{r}$ в потеншиале

$$
V=a^{2}\left(x_{1} x_{2} x_{3}\right)^{-2 / 3}, \quad a \in \mathbb{R} .
$$

Данная двумерная динамическая система является интегрируемой по Лиувиллю, т.е. обладает вторым интегралом движения [5]. Более того, уравнения движения были проинтегрированы в квадратурах после замены времени и неканонического преобразования координат [6].

\section{2. УРАВНЕНИЯ ДВИЖЕНИЯ В ФОРМЕ ЛАКСА}

Рассмотрим движение точки по поверхности сферы $S^{2}$ и отождествим конфигурационное пространство данной системы с группой $E(3)$ движений трехмерного евклидова пространства, а фазовое пространство - с кокасательным пространством $T^{*} E(3)$ [7]. При этом кинетическая энергия является левоинвариантной римановой метрикой на $T^{*} E(3)$, которая определяется весьма специальной квадратичной формой на пространстве $e^{*}(3)$, двойственном к алгебре Ли $e(3)[7,8]$.

Используя форму Киллинга, отождествим дуальное пространство $e^{*}(3)$ с пространством $e(3)=s o(3) \oplus \mathbb{R}^{3}$, которое является полупрямой суммой $s o(3)$ и абелева идеала $\mathbb{R}^{3}$. Пусть два трехмерных вектора $J \in s o(3) \simeq \mathbb{R}^{3}$ и $x \in \mathbb{R}^{3}$ являются координатами в пространстве $e^{*}(3)$, снабженном естественной скобкой Ли-Пуассона

$$
\left\{J_{i}, J_{j}\right\}=\varepsilon_{i j k} J_{k}, \quad\left\{J_{i}, x_{j}\right\}=\varepsilon_{i j k} x_{k}, \quad\left\{x_{i}, x_{j}\right\}=0,
$$

где $\varepsilon_{i j k}$ - стандартный полностью кососимметричный тензор. Представления алгебры $e(3)$ фиксируются значениями двух операторов Казимира

$$
\mathbf{C}_{1}=(x, x)=x_{i} x_{i}, \quad \mathbf{C}_{2}=(J, x)=J_{i} x_{i} .
$$

Орбиты $\mathcal{O}\left(\mathbf{C}_{1}=c_{1}, \mathbf{C}_{2}=c_{2}\right)$ коприсоединенного представления $E(3)$ в $e(3)^{*}$ являются четырехмерными симплектическими многообразиями, инвариантными относительно уравнений Эйлера-Пуассона $[7,8]$. Данные многообразия топологически эквивалентны кокасательному расслоению $T^{*} S^{2}$ к двумерной сфере $S^{2}$.

Введем комплексный аналог вещественной алгебры $e(3)$, используя полупрямую сумму алгебры $s o(3, \mathbb{C})$ и комплексного абелевого идеала $\mathbb{C}^{3}$. Для полученной таким образом алгебры $e(3, \mathbb{C})=s o(3, \mathbb{C}) \oplus \mathbb{C}^{3}$, как и ранее, зададим те же скобки Ли-Пуассона $(2.1)$ и операторы Казимира (2.2). Переход к комплексному абелеву идеалу позволяет рассмотреть нетривиальные представления данной алгебры с нулевым значением $c_{1}=0$ первого оператора Казимира $\mathbf{C}_{1}$.

Условие $c_{1}=0$ не имеет физического или геометрического смысла, т.к. означает, что мы рассматриваем движение по поверхности сферы нулевого радиуса. С математической точки зрения такие представления комплексной алгебры вполне допустимы. Заметим, что подобные, на первый взгляд "не физические", представления алгебры $s l(2)$ с нулевым спином $s=0$ уже используются в различных областях физики $[9,10]$. 
ПРеДЛОЖЕнИЕ 1. Отображение подалгебры so* $^{*}(3, \mathbb{C}) \subset e^{*}(3, \mathbb{C})$

$$
J \rightarrow \widetilde{J}=J+\frac{i a}{\left(x_{1} x_{2} x_{3}\right)^{1 / 3}} x, \quad a \in \mathbb{C},
$$

является внешним автоморфизмом представления комплексной алгебры е $(3, \mathbb{C})$ с нулевым значением $c_{1}=0$ первого оператора Казимира $\mathbf{C}_{1}(2.2)$.

Используя вложение $e(3) \subset e(3, \mathbb{C})$, любой из известных интегрируемых волчков мы можем рассмотреть как динамическую систему на комплексном фазовом пространстве $e^{*}(3, \mathbb{C})$. Далее, применяя отображение $(2.3)$, построим деформации данных интегрируемых волчков, которые будут так же интегрируемыми системами на однопараметрическом множестве орбит $\mathcal{O}_{1}\left(c_{1}=0, c_{2}=\right.$ const). Однако, как мы покажем ниже, данная процедура позволяет получить и другие интегрируемые обобщения.

Рассмотрим шаровый волчок, определяемьй функцией Гамильтона вида

$$
H=(\widetilde{J}, \widetilde{J})=\widetilde{J}_{1}^{2}+\widetilde{J}_{2}^{2}+\widetilde{J}_{3}^{2}
$$

и (не совсем обычным) вторым интегралом движения

$$
K=\widetilde{J}_{1} \widetilde{J}_{2} \widetilde{J}_{3} .
$$

Заметим, что оба интеграла движения определены только на подалгебре $s o(3)$. Применяя отображение (2.3), мы получаем обобщение шарового волчка, интегрируемое при $c_{1}=0$.

ПРЕДЛОЖЕНИЕ 2. Внешний автоморфизм (2.3) переводит функиию Гамильтона шарового волчка (2.4) в функцию Гамильтона вида

$$
H=\sum_{k=1}^{3} J_{k}^{2}+2 i a \frac{c_{2}}{\left(x_{1} x_{2} x_{3}\right)^{1 / 3}}-a^{2} \frac{c_{1}}{\left(x_{1} x_{2} x_{3}\right)^{2 / 3}} .
$$

Динамическая система с данным гамильтонианом является вполне интегрируемой на двух возможных множествах однопараметрических орбит

$$
\mathcal{O}_{1}:\left(c_{1}=0, c_{2}=\text { const }\right) \quad u \quad \mathcal{O}_{2}:\left(c_{1}=\text { const }, c_{2}=0\right) .
$$

Динамическая система на втором множестве орбит $\mathcal{O}_{2}$ остается интегрируемой и при переходе к обичной вещественной алгебре $e^{*}(3)$.

Заметим, что при $c_{2}=0$ построенная нами функция Гамильтона совпадает с гамильтонианом (1.2), описываюшим расширение облака идеального одноатомного газа.

При $c_{1}=0$ интеграл шарового волчка $K(2.5)$ является прообразом нового дополнительного второго интеграла вида

$$
\begin{aligned}
K= & J_{1} J_{2} J_{3}-a^{2}\left(\frac{J_{1}}{x_{1}}+\frac{J_{2}}{x_{2}}+\frac{J_{3}}{x_{3}}\right)\left(x_{1} x_{2} x_{3}\right)^{1 / 3}+ \\
& +\frac{2 i a}{\left(x_{1} x_{2} x_{3}\right)^{1 / 3}}\left(J_{1} J_{2} x_{3}+J_{1} x_{2} J_{3}+x_{1} J_{2} J_{3}\right)-i a^{3} .
\end{aligned}
$$


При $c_{2}=0$ дополнительный второй интеграл движения [5] есть

$$
K=J_{1} J_{2} J_{3}+a^{2}\left(\frac{J_{1}}{x_{1}}+\frac{J_{2}}{x_{2}}+\frac{J_{3}}{x_{3}}\right)\left(x_{1} x_{2} x_{3}\right)^{1 / 3} .
$$

По сравнению с интегралом (2.7) здесь отсутствуют мнимые слагаемые и изменен знак перед единственным оставшимся потенциальным слагаемым. То, что при переходе к вещественной алгебре мы отбросили мнимые слагаемые, выглядит достаточно естественно. Причину замены знака мы объяснить не можем.

В новых переменных

$$
y=\frac{x}{\left(x_{1} x_{2} x_{3}\right)^{1 / 3}}, \quad y_{1} y_{2} y_{3}=1,
$$

которые удобно использовать для данной системы, отображение (2.3) становится просто сдвигом $\widetilde{J}=J+i a y$, а интегралы движения (2.6) и (2.8) становятся похожими на интегралы шарового волчка в однородном квадратичном потенциале:

$$
\begin{aligned}
& H=J_{1}^{2}+J_{2}^{2}+J_{3}^{2}-a^{2}\left(y_{1}^{2}+y_{2}^{2}+y_{3}^{2}\right), \\
& K=J_{1} J_{2} J_{3}+a^{2}\left(\frac{J_{1}}{y_{1}}+\frac{J_{2}}{y_{2}}+\frac{J_{3}}{y_{3}}\right) .
\end{aligned}
$$

Порождаемые гамильтонианом (2.6) уравнения Эйлера-Пуассона в пространстве $e^{*}(3)$ имеют вид

$$
\begin{aligned}
\frac{d}{d t} J & =\frac{2 a^{2}}{3}(y, y) y \times y^{-1}, & y^{-1} & =\left(y_{1}^{-1}, y_{2}^{-1}, y_{3}^{-1}\right), \\
\frac{d}{d t} y & =-\frac{2}{3}(y, y) J \times y^{-1}, & (y, y) & =y_{1}^{2}+y_{2}^{2}+y_{3}^{2} .
\end{aligned}
$$

Здесь $x \times y$ - обычное векторное произведение трехмерных векторов в пространстве $\mathbb{R}^{3}$. Таким образом, мы переписали исходные симметричные уравнения движения (1.4) в конфигурационном пространстве $\mathbb{R}^{3}$ в виде уравнений Эйлера-Пуассона $(2.10)$ в фазовом пространстве $e^{*}(3)$.

Данные уравнения движения (2.10) мы постараемся записать в форме уравнений Лакca

$$
\frac{d}{d t} L=[L, M]
$$

Поэтому мы кратко обсудим обший подход к построению матриц Лакса [8] в рамках метода классической $r$-матрицы на примере системы Неймана, которая описывает движение по поверхности сферы в поле квадратичного потенциала:

$$
V=\left(a_{1} x_{1}^{2}+a_{2} x_{2}^{2}+a_{3} x_{3}^{2}\right), \quad a_{i} \in \mathbb{R}
$$

Фазовое пространство системы отождествим с однопараметрическим множеством орбит $e^{*}(3)$ при $c_{2}=0$. Уравнения Эйлера-Пуассона имеют вид

$$
\frac{d}{d t} J=x \times z, \quad \frac{d}{d t} x=-J \times x, \quad z=-\operatorname{diag}\left(a_{1}, a_{2}, a_{3}\right) x
$$


здесь $a_{j}$ - произвольные параметры.

В качестве полного набора интегралов, находяшихся в инволюции, можно выбрать интегралы Уленбека

$$
I_{j}=\frac{1}{2}\left(p_{j}^{2}+a_{j} x_{j}^{2}\right)+\sum_{k} \frac{\left(p_{j} x_{k}-p_{k} x_{j}\right)^{2}}{a_{j}-a_{k}} .
$$

Данные интегралы являются квадратичными функциями от импульсов. Тем не менее даже такие интегралы не могут быть получены с помошью квадратичных инвариантных функций на алгебре $e(3)=s o(3) \oplus \mathbb{R}^{3}$. В этом случае обычно рассматривают более широкую алгебру, в которую исходная алгебра вложена в качестве подалгебры. Действительно, для описания системы Неймана и некоторых других волчков используют разложение Картана алгебры $g l(3, \mathbb{R})=s o(3)+\operatorname{Symm}(3)[8]$.

Итак, вводя кососимметрическую матрицу компонент углового момента $\mathcal{J} \in s o(3)$ и симметрическую матрицу координат $\mathcal{X} \in \operatorname{Symm}(3)$,

$$
\begin{array}{ll}
\mathcal{J} \in \operatorname{so}(3): & \mathcal{J}_{i j}=\varepsilon_{i j k} \mathcal{J}_{k}=p \times x, \\
\mathcal{X} \in \operatorname{Symm}(3): & \mathcal{X}_{i j}=x_{i} x_{j},
\end{array}
$$

получим следующее представление Лакса для системы Неймана:

$$
L=\operatorname{diag}\left(a_{1}, a_{2}, a_{3}\right) \lambda+\mathcal{J}+\lambda^{-1} \mathcal{X}, \quad M=-\lambda^{-1} \mathcal{X} .
$$

Вьпишем эти матрицы в явном виде

$$
\begin{gathered}
L=\left(\begin{array}{ccc}
a_{1} \lambda & 0 & 0 \\
0 & a_{2} \lambda & 0 \\
0 & 0 & a_{3} \lambda
\end{array}\right)+\left(\begin{array}{ccc}
0 & -J_{3} & J_{2} \\
J_{3} & 0 & -J_{1} \\
-J_{2} & J_{1} & 0
\end{array}\right)+\frac{1}{\lambda}\left(\begin{array}{ccc}
x_{1}^{2} & x_{1} x_{2} & x_{1} x_{3} \\
x_{1} x_{2} & x_{2}^{2} & x_{2} x_{3} \\
x_{1} x_{3} & x_{2} x_{3} & x_{3}^{2}
\end{array}\right), \\
M=-\frac{x_{1} x_{2} x_{3}}{\lambda}\left(\begin{array}{ccc}
\frac{x_{1}}{x_{2} x_{3}} & x_{3}^{-1} & x_{2}^{-1} \\
x_{3}^{-1} & \frac{x_{2}}{x_{1} x_{3}} & x_{1}^{-1} \\
x_{2}^{-1} & x_{1}^{-1} & \frac{x_{3}}{x_{1} x_{2}}
\end{array}\right) .
\end{gathered}
$$

Вернемся теперь к рассмотрению движения по сфере с функцией Гамильтона (2.6) и кубическим по импульсам дополнительным интегралом движения. Напомним, что пространство $\mathbb{R}^{3}$ с заданным векторным произведением и алгебра Ли so(3) с обычными правилами коммутации могут быть отождествлены:

$$
x=\left(\begin{array}{l}
x_{1} \\
x_{2} \\
x_{3}
\end{array}\right) \in \mathbb{R}^{3} \longrightarrow \mathcal{X}=\left(\begin{array}{ccc}
0 & x_{3} & -x_{2} \\
-x_{3} & 0 & x_{1} \\
x_{2} & -x_{1} & 0
\end{array}\right) \in s o(3) .
$$

Данное отображение является известным изоморфизмом алгебр Ли. Заметим, что в отображении $(2.3)$ к элементу $J \in s o(3)$ добавляется вектор $x \in \mathbb{C}^{3}$, т.е., определяя 
внешний автоморфизм пространства представлений (2.3), мы уже неявно использовали изоморфизм алгебр Ли, который имеет место только в трехмерном пространстве. Поэтому нам не удалось найти обобшений предложенного внешнего автоморфизма (2.3) для $n$-мерного пространства $\mathbb{C}^{n}$ и соответствующих интегрируемых движений по $(n-$ 1)-мерной сфере $S^{n-1}$ при $n>3$.

При построении матрицы Лакса для деформации шарового волчка переставим слагаемые в разложении Картана $g l(3, \mathbb{R})=s o(3)+\operatorname{Symm}(3)$, используя изоморфизм алгебр Ли (некий аналог преобразования Фурье в квантовой механике). Итак, введем симметрическую матрицу углового момента $\mathcal{J} \in \operatorname{Sym}(3)$ и кососимметрическую матрицу координат $\mathcal{X} \in s o(3)$,

$$
\begin{array}{ll}
\mathcal{J} \in \operatorname{Symm}(3): & \mathcal{J}_{i j}=\left|\varepsilon_{i j k}\right| J_{k}, \\
\mathcal{X} \in \operatorname{so}(3) \simeq \mathbb{R}^{3}: & \mathcal{X}_{i j}=\varepsilon_{i j k} y_{k}
\end{array}
$$

где $\left|\varepsilon_{i j k}\right|$ - абсолютное значение кососимметрического тензора $\varepsilon_{i j k}$.

ПРЕДЛОЖЕНИЕ 3. При $c_{2}=0$ уравнения Эйлера-Пуассона (2.10), описъвающие движение по поверхности сферь $S^{2}$ с функиией Гамильтона (2.9), могут быть записаны в форме Лакса (2.11) с матрицами вида

$$
L=\lambda I+\mathcal{J}+a \mathcal{X}, \quad M_{i j}=\frac{2 a}{3}\left|\varepsilon_{i j k}\right| x_{k}^{-1},
$$

$2 \partial e$

$$
L=\left(\begin{array}{ccc}
\lambda & 0 & 0 \\
0 & \lambda & 0 \\
0 & 0 & \lambda
\end{array}\right)+\left(\begin{array}{ccc}
0 & J_{3} & J_{2} \\
J_{3} & 0 & J_{1} \\
J_{2} & J_{1} & 0
\end{array}\right)+a\left(\begin{array}{ccc}
0 & y_{3} & -y_{2} \\
-y_{3} & 0 & y_{1} \\
y_{2} & -y_{1} & 0
\end{array}\right)
$$

$u$

$$
M=\frac{2 a c_{1}^{1 / 2}}{3 \sqrt{(y, y)}}\left(\begin{array}{ccc}
0 & y_{3}^{-1} & y_{2}^{-1} \\
y_{3}^{-1} & 0 & y_{1}^{-1} \\
y_{2}^{-1} & y_{1}^{-1} & 0
\end{array}\right)
$$

Характеристический полином первой из матрии является порождающей функиией полного набора интегралов движения в инволюиии (2.9)

$$
\operatorname{det} L(\lambda)=\lambda^{3}-H \lambda+2 K
$$

В формуле для $L$ постоянную диагональную матрицу, которая зависит от спектрального параметра, мы добавили только для аналогии с системой Неймана. В действительности предлагаемая матрица Лакса не зависит от спектрального параметра и соответствуюшая спектральная кривая $\operatorname{det}(\mu I+L)$ вырождена. Данный недостаток не позволяет использовать известные алгебро-геометрические методы для интегрирования уравнений движения. Однако и такая матрица Лакса может быть полезна при поиске представления Лакса со спектральным параметром. Напомним, например, что матрица Лакса без спектрального параметра, предложенная Переломовым для волчка Ковалевской, является элементом более полного представления Лакса со спектральным параметром [8]. 


\section{3. МЕТОД КОВАЛЕВСКОЙ-ПЕНЛЕВЕ}

Уравнения движения интегрируемой системы на сфере с гамильтонианом (2.6) были проинтегрированы в квадратурах в работе [6]. Предложенное в [6] неканоническое преобразование переменных зависит от энергии системы, но зато переменные разделения лежат на гиперэллиптической кривой второго рода. Процедура построения данного преобразования явно зависит от конкретного вида уравнений движения и близка к исследованию Чаплыгина [11], посвяшенному уравнениям Кирхгофа.

В данной работе мы применим более общий подход, основанный на методе Ковалевской-Пенлеве [12-14], который не столь явно зависит от вида уравнений движения. Напомним, что данный подход успешно использовался для интегрирования уравнений движения систем Неймана и Энона-Эйлеса, цепочки Тоды, волчков Горячева-Чаплыгина и Ковалевской и для некоторых других интегрируемых систем [12-14]. Все эти конкретные системы связаны с гиперэллиптическими алгебраическими кривыми.

Напомним, что этот более обший метод можно использовать только для систем уравнений движения однородного веса [12-14]. Обычно уравнения движения, записанные в исходных физических координатах, не удовлетворяют данному требованию. Поэтому необходимо ввести новые координаты так, что соответствуюшие уравнения движения будут удовлетворять необходимым условиям. Например, для интегрирования цепочки Тоды необходимо перейти от физических переменных к переменным Флашки. Построение таких переменных является отдельной задачей, которая пока не имеет обшего решения. Тем не менее, если удается найти требуемую систему координат, то применение метода Ковалевской-Пенлеве обычно позволяет проинтегрировать данную систему уравнений [12-14].

Начнем с трехчастичной периодической цепочки Тоды с функцией Гамильтона

$$
H=\frac{1}{2} \sum_{j=1}^{3} p_{j}^{2}+e^{q_{1}-q_{2}}+e^{q_{2}-q_{3}}+e^{q_{3}-q_{1}} .
$$

Здесь $p_{j}, q_{j}$ - пары канонически-сопряженных переменных, $\left\{p_{j}, q_{k}\right\}=\delta_{j k}$. Следуя работам [12-14], введем переменные Флашки

$$
\begin{array}{ll}
z_{1}=e^{q_{1}-q_{2}}, & z_{2}=e^{q_{2}-q_{3}}, \\
z_{4}=-p_{1}, & z_{3}=e^{q_{3}-q_{1}}, \quad p_{2}, \quad z_{2} z_{3}=1, \\
z_{6}=-p_{3}
\end{array}
$$

и перепишем уравнения движения в виде

$$
\dot{z}_{j}=f_{j}\left(z_{1}, \ldots, z_{n}\right), \quad j=1, \ldots, n,
$$

где

$$
\begin{array}{ll}
\frac{d}{d t} z_{1}=z_{1}\left(z_{5}-z_{4}\right), & \frac{d}{d t} z_{4}=z_{1}-z_{3}, \\
\frac{d}{d t} z_{2}=z_{2}\left(z_{6}-z_{5}\right), & \frac{d}{d t} z_{5}=z_{2}-z_{1}, \\
\frac{d}{d t} z_{3}=z_{3}\left(z_{4}-z_{6}\right), & \frac{d}{d t} z_{6}=z_{3}-z_{2} .
\end{array}
$$


Данная система уравнений является системой однородного веса, т.е. сушествуют положительные целые веса $\nu_{j}$ такие, что для любого $\lambda \in \mathbb{C}$ справедливы равенства

$$
f_{j}\left(\lambda_{1}^{\nu} z_{1}, \ldots, \lambda_{n}^{\nu} z_{n}\right)=\lambda^{\nu_{j+1}} f_{j}\left(z_{1}, \ldots, z_{n}\right) .
$$

Для цепочки Тоды вес переменных Флашки $z_{1}, z_{2}, z_{3}$ равен $\nu_{123}=2$, а переменных $z_{4}$, $z_{5}, z_{6}$ равен $\nu_{456}=1$. Соответствуюшие интегралы движения

$$
\begin{aligned}
& Z_{1}=z_{1} z_{2} z_{3}=1, \\
& Z_{2}=z_{4}+z_{5}+z_{6}=d_{1}=0, \\
& Z_{3}=\frac{1}{2}\left(z_{4}^{2}+z_{5}^{2}+z_{6}^{2}\right)+z_{1}+z_{2}+z_{3}=a_{1}, \\
& Z_{4}=z_{4} z_{5} z_{6}-z_{1} z_{6}-z_{2} z_{4}-z_{3} z_{5}=b_{1}
\end{aligned}
$$

являются функциями веса $\{6,1,2,3\}$ соответственно.

Уравнения движения для цепочки Тоды инвариантны относительно действия группы циклических перестановок

$$
q \longmapsto \mathcal{D} q, \quad p \longmapsto \mathcal{D} p, \quad \mathcal{D}=\left(\begin{array}{lll}
0 & 1 & 0 \\
0 & 0 & 1 \\
1 & 0 & 0
\end{array}\right), \quad \mathcal{D}^{3}=1 .
$$

Таким образом, инвариантное многообразие (3.3) обладает автоморфизмом третьего порядка

$$
\left(z_{1}, z_{2}, z_{3}, z_{4}, z_{5}, z_{6}\right) \longmapsto\left(z_{2}, z_{3}, z_{1}, z_{5}, z_{6}, z_{4}\right),
$$

который упрошает анализ Пенлеве системы уравнений движения $[12,14]$.

Вернемся теперь к интегрируемой деформации шарового волчка (2.6). Уравнения движения (2.10) удовлетворяют слабому критерию Ковалевской-Пенлеве, т.е. решения уравнений движения допускают особенности типа $\left(t-t_{0}\right)^{m / k}$, где $m$ и $k$ - целые числа. Напомним, что в обычном условии Ковалевской-Пенлеве в решениях уравнений допускается существование только особенностей типа полюса. Поэтому, следуя обычному в данных случаях рецепту [12], перейдем к новому времени и новым скобкам Пуассона по правилу

$$
t \rightarrow u: \quad \frac{d}{d u}=\frac{4}{3}(y, y) \frac{d}{d t}, \quad\{\cdot, \cdot\}_{1}=\frac{4}{3}(y, y)\{\cdot, \cdot\}_{0} .
$$

Уравнения Эйлера-Пуассона (2.10) относительно нового времени имеют вид

$$
\frac{d}{d u} J=\frac{a^{2}}{2} y \times y^{-1}, \quad \frac{d}{d u} y=-\frac{1}{2} J \times y^{-1} .
$$

Именно эти уравнения были проинтегрированы в гиперэллиптических квадратурах в работе [6]. Однако переменные разделения $\lambda_{1,2}$ зависят друг от друга, и скобка Пуассона между ними не равна нулю $\left\{\lambda_{1}, \lambda_{2}\right\} \neq 0$. 
Система (3.7) не является системой однородного веса, т.к. соответствующие уравнения (3.2) не имеют решений. Поэтому вместо исходных переменных нам необходимо ввести новые переменные. Как уже отмечалось выше, обших методов построения переменных с заданными свойствами не известно. Однако аналог переменных Флашки для движения по поверхности сферы $S^{2}$ можно построить, заметив, что уравнения движения (3.1) для цепочки Тоды инвариантны относительно следуюшего отображения:

$$
z_{j} \longmapsto \frac{1}{z_{j}}, \quad z_{j+3} \longmapsto-z_{j+3}, \quad j=1,2,3 .
$$

Первое из уравнений Эйлера-Пуассона (3.7) также инвариантно относительно подобного преобразования,

$$
y_{j} \longmapsto \frac{1}{y_{j}}, \quad J_{j} \longmapsto-J_{j}, \quad j=1,2,3 .
$$

Используя этот факт, введем новые переменные $\left\{s_{j}\right\}_{j=1}^{6}$ по правилу

$$
\begin{aligned}
& s_{1}=y_{1}^{-2}, \quad s_{2}=y_{3}^{-2}, \quad s_{3}=y_{2}^{-2}, \quad s_{1} s_{2} s_{3}=1, \\
& s_{6}=y_{1} J_{1}, \quad s_{4}=y_{3} J_{3}, \quad s_{5}=y_{2} J_{2} \text {. }
\end{aligned}
$$

Система соответствующих уравнений движения

$$
\begin{aligned}
\frac{d}{d u} s_{1} & =s_{1}\left(s_{5}-s_{4}\right), & \frac{d}{d u} s_{4} & =\frac{a^{2}}{2}\left(s_{3}-s_{1}\right)+\frac{s_{4}}{2}\left(s_{5}-s_{6}\right), \\
\frac{d}{d u} s_{2} & =s_{2}\left(s_{6}-s_{5}\right), & \frac{d}{d u} s_{5} & =\frac{a^{2}}{2}\left(s_{1}-s_{2}\right)+\frac{s_{5}}{2}\left(s_{6}-s_{4}\right), \\
\frac{d}{d u} s_{3} & =s_{3}\left(s_{4}-s_{6}\right), & \frac{d}{d u} s_{6} & =\frac{a^{2}}{2}\left(s_{2}-s_{3}\right)+\frac{s_{6}}{2}\left(s_{4}-s_{5}\right)
\end{aligned}
$$

является однородной по весу. Более того, уравнения из первого столбца совпадают с соответствуюшими уравнениями для цепочки Тоды (3.1), а уравнения из второго столбца отличаются одним добавочным слагаемым. Таким образом, данную интегрируемую систему можно рассматривать как интегрируемое обобшение цепочки Тоды.

Как и для цепочки Тоды, вес новых переменных $s_{1}, s_{2}, s_{3}$ равен $\nu_{123}=2$, а переменных $s_{4}, s_{5}, s_{6}$ равен $\nu_{456}=1$. При этом интегралы движения

$$
\begin{aligned}
& S_{1}=s_{1} s_{2} s_{3}=1, \\
& S_{2}=s_{4}+s_{5}+s_{6}=0, \\
& S_{3}=\left(s_{4}^{2} s_{2}+s_{5}^{2} s_{3}+s_{6}^{2} s_{1}\right)-a^{2}\left(s_{1} s_{2}+s_{1} s_{3}+s_{2} s_{3}\right)=a_{1}, \\
& S_{4}=s_{4} s_{5} s_{6}+a^{2}\left(s_{1} s_{6}+s_{2} s_{4}+s_{3} s_{5}\right)=b_{1}
\end{aligned}
$$

являются функциями веса $\{6,1,4,3\}$ соответственно. Более того, интегралы $S_{1}, S_{2}$ и $S_{4}$ совпадают с интегралами для цепочки Тоды при $a^{2}=-1$. Вес функции Гамильтона 
$S_{3}$ меняется с 2 до 4, тогда как сама функция является кубическим симметрическим полиномом.

Как и для цепочки Тоды, уравнения движения (3.9) инвариантны относительно действия группы циклических перестановок (3.4), а инвариантное многообразие (3.10) обладает автоморфизмом третьего порядка (3.5).

Применение данного автоморфизма упрощает пенлеве-анализ и позволяет построить одновременно три семейства лорановских разложений вида

$$
s_{i}=t^{-2}\left(s_{i}^{(0)}+s_{i}^{(1)}+\cdots\right), \quad s_{i+3}=t^{-1}\left(s_{i+3}^{(0)}+s_{i+3}^{(1)}+\cdots\right), \quad i=1,2,3,
$$

где мы явно подставили веса $\nu_{j}$ переменных $s_{j}$. Следуя [12], далее необходимо построить такие разложения, которые зависят от максимального $n-1$ числа свободных параметров. В нашем случае решения должны зависеть от пяти параметров. Первые коэффициенты разложений находятся из уравнений

$$
\nu_{i} s_{i}^{(0)}+f_{i}\left(s^{(0)}\right)=0, \quad i=1, \ldots, 6 .
$$

Матрица Якоби данной системы уравнений

$$
K_{i j}=\frac{\partial f_{i}}{\partial z_{j}}+\delta_{i j} \nu_{j}
$$

называется матрицей Ковалевской, а ее собственные значения - экспонентами Ковалевской.

Система уравнений для цепочек Тоды (3.1) и новая система уравнений (3.9) обладают одним обшим семейством решений уравнений (3.12) при $a=1$. Спектр матрищы Ковалевской также не меняется и равен $\{-1,1,1,2,3,3\}$. Таких решений три в соответствии с инвариантностью относительно перестановок. Именно эти решения порождают лорановские разложения (3.11), зависящие от максимального числа параметров (принципиальные балансы). Для цепочки Тоды сушествует еше одно семейство решений. Для волчка можно построить два дополнительных семейства решений. Соответствуюшие разложения зависят только от трех свободных параметров, и спектр матриц Ковалевской имеет вид $\{-2,-1,1,3,4,4\}$ и $\{-4,-1,1,3,4,6\}$. Такие решения называются низшими (или вторичными) балансами системы уравнений движения.

Остальные коэффициенты лорановских рядов (3.11) находятся рекурсивно как решения уравнений

$$
\left[K\left(s^{(0)}\right)-m I\right] s^{(m)}=Y, \quad m=1,2, \ldots
$$

Здесь $Y$ - векторный полином, составленный из найденных ранее векторов $s^{(0)}, \ldots$ $\ldots, s^{(m-1)}[12]$. Выпишем явно три первых коэффициента в рядах $(3.11)$ для одного из принципиальных балансов:

$$
\begin{array}{lccc} 
& \left(s_{1}^{(0)}, \ldots, s_{6}^{(0)}\right) & \left(s_{1}^{(1)}, \ldots, s_{6}^{(1)}\right) & \left(s_{1}^{(2)}, \ldots, s_{6}^{(2)}\right) \\
\text { Цепочка Тоды } & \{1,0,0,1,-1,0\} & \{0,0,0, \alpha, \alpha, \beta\} & \{-\gamma, 0,0,-\gamma, \gamma, 0\} \\
\text { Волчок } & \left\{a^{-2}, 0,0,1,-1,0\right\} & \left\{\alpha, 0,0,-a^{2} \alpha+\beta, \beta, 0\right\} & \{0,0,0, \gamma, \gamma,-2 \gamma\}
\end{array}
$$


Здесь $\alpha, \beta$ и $\gamma-$ свободные параметры. Только первый коэффициент совпадает для рядов, отвечаюших цепочке Тоды и движению по поверхности сфферы. Остальные коэффициенты рядов различны.

Подставляя лорановские ряды (3.11) в определение инвариантного многообразия (3.10) и решая возникаюшие уравнения, получаем объединение алгебраических кривых $\mathcal{C}_{1} \cup \mathcal{C}_{2} \cup \mathcal{C}_{3}$ (дивизор Пенлеве). Как и для цепочек Тоды, для волчка все три кривые $\mathcal{C}_{j}$ одинаковы и изоморфны кривой

$$
\mu^{2}\left[\left(\mu+b_{1}\right)^{2}+4 a^{6}\right]-\lambda^{2}\left(\lambda^{2}-a^{2} a_{1}\right)^{2}=0 .
$$

Напомним, что для цепочек Тоды подобная процедура приводит к гиперэллиптической кривой [14] вида

$$
\mu-b_{1}+\mu^{-1}=\lambda\left(\lambda^{2}-a_{1}\right) .
$$

Однако, если для цепочки Тоды вместо интеграла движения $Z_{3}(3.3)$ использовать интеграл $Z_{3}^{2}$ веса 4 , равного весу интеграла $S_{3}$ для волчка, мы получим более сложную кривую

$$
\left(\mu-b_{1}+\mu^{-1}\right)^{2}+2 \lambda^{3}\left(\mu-b_{1}+\mu^{-1}\right)+\lambda^{2}\left(\lambda^{4}-a_{1}^{2}\right)=0 .
$$

Переменные разделения, связанные с семействами интегралов $\left\{Z_{3}, Z_{4}\right\}$ и $\left\{Z_{3}^{2}, Z_{4}\right\}$, различны, хотя уравнения движения должны линеаризоваться в обоих случаях. Поэтому алгебраические кривые (3.15) и (3.16), по-видимому, также различны, хотя и имеют общие свойства.

Итак, мы построили алгебраическую кривую (3.14), связанную с семейством интегралов $\left\{S_{3}, S_{4}\right\}$ (3.10). Далее по общей схеме необходимо описать сингулярности инвариантного многообразия (3.10), пополнить подходящим образом данное многообразие до аффинной части абелева многообразия и описать вложение этого пространства в подходящее проективное пространство $[12,14]$.

К сожалению, все ранее известные примеры и разработанные для их решения методы применимы в основном только к гиперэллиптическим кривым. Для рассматриваемой нами интегрируемой системы, связанной с более сложной алгебраической кривой, известные методы непосредственно неприменимы. Например, для цепочки Тоды сушествуют две переменные $z_{6}=$ const $+O(t)$ и $z_{7}=z_{4} z_{5}-z_{1}=$ const $+O(t)$, которые можно использовать для регуляризации $[12,14]$ уравнений движения одновременно для гиперэллиптической кривой (3.15) и кривой (3.16). Для движения по поверхности сферы нам удалось найти только одну необходимую переменную $\left(s_{4}-s_{5}\right) s_{6}=$ const $+O(t)$, обладающую таким свойством.

Изучение данной интегрируемой деформации шарового волчка может быть продолжено либо в рамках алгебраической геометрии с использованием кривой вида (3.14), либо в рамках метода классической $r$-матрицы с использованием матрицы Лакса со спектральным параметром, связанной с некоторой алгеброй Ли $g^{*}$. Заметим, что алгебраические кривые, полученные в результате применения этих двух методов, могут быть различными, как в случае волчка Ковалевской $[8,12]$.

6 Теоретическая и математическая физика, т. 124, № 2, 2000 г. 


\section{Список литературы}

[1] Л. В. Овсянников. ДАН СССР. 1956. Т. 111. С. 47.

[2] F. J. Dyson. J. Math. Mech. 1968. V. 18. P. 91.

[3] С.И. Анисимов, И. И. Льсиков. ПММ. 1970. Т. 34. С. 926.

[4] О.И. Богоявленский. Методы качественной теории динамических систем в астрофизике и газовой динамике. М.: Наука, 1980.

[5] B. Gaffet. J. Fluid Mech. 1996. V. 325. P. 113.

[6] B. Gaffet. J. Phys. A. 1998. V. 31. P. 1581; P. 8341.

[7] В. И. Арнольд. Математические методы классической механики. М.: Наука, 1979.

[8] Итоги науки и техники. Современные проблемы математики. Фунд. направления. Динамические системы-7. Т. 16. Ред. Р. В Гамкрелидзе. М.: ВИНИТИ, 1987.

[9] L. D. Faddeev, G. P. Korchemsky. Phys. Lett. B. 1995. V. 342. P. 311.

[10] А. В Цыганов. ТМФ. 1999. Т. 118. С. 205.

[11] С. А. Чапльљин. Собрание сочинений. Т. 1-2. М.: Гостехиздат, 1948.

[12] M. Adler, P. van Moerbeke. Invent. Math. 1989. V. 97. P. 3; Algebraic completely integrable systems: A systematic approach. New York: Academic Press, 1993.

[13] C. Bechlivanidis, P. van Moerbeke. Commun. Math. Phys. 1987. V. 110. P. 317.

[14] P. Vanhaecke. Math. Z. 1992. V. 211. P. 265.

Поступила в редакцию 5.XI.1999 г. 